\title{
Stability and Convergence of a Finite Volume Method for Two Systems of Reaction-Diffusion Equations in Electro-Cardiology
}

\author{
Yves Coudière Charles Pierre \\ Laboratoire de Mathématiques Jean Leray, Nantes University and CNRS - UMR 6629, \\ France
}

\begin{abstract}
The monodomain equations model the propagation of the action potential in the human heart : a very sharp pulse propagating at a high speed, which computation require fine unstructured 3D meshes. It is a non linear parabolic PDE of reaction diffusion type, coupled to one or several ODE, with multiple time-scales.

Numerical difficulties, such as unstructured meshes and stability are addressed here through the use of a finite volume method. Stability conditions are given for two timestepping methods, and two example sets of ODEs, convergence is proved and error estimates are computed.
\end{abstract}

Key words:

\section{Introduction}

Computer models of the electrical activity in the myocardium are increasingly popular : the heart's activity generates an electromagnetic field in the torso, and produces a surface potential map which measure is the well-known electrocardiogram (ECG). It gives a non-invasive representation of the cardiac electrical function.

This paper focuses on the study of a 3D finite volume numerical method used to compute the electrical activity of the myocardium on unstructured meshes, and specifically gives conditions on the time-step to ensure a $L^{\infty}$ stability property, for an explicit and a semi-implicit time-stepping method. Consequently, convergence results are proved.

The electrical activity on the torso was first demonstrated to be directly connected to the heart beat more than 100 years ago [26]. It was first suggested to be well 
represented by a dipole. Afterward, more complex models based on dipole representation have also been used among which the famous oblique dipole layer [5]. This is the top-down approach, providing heuristic models.

Conversely, in the 50's Hodgkin and Huxley [11] explained how the electrical activity of some nerve cells can be modeled from a microscopic description of ionic currents through the membrane. Due to the sophistication of experimental techniques, there are currently many such models, see [12] for reviews.

Recent studies in electrocardiology assume the anisotropic cardiac tissue to be represented at a macroscopic level by the so-called "bidomain" model, despite the discrete structure of the tissue. We refer to [8] for a mathematical derivation of the bidomain equations, and to $[9,12]$ for reviews on the bidomain equations. A simpler version called the "monodomain" model is obtained, assuming an additional condition on the anisotropy of the tissue. Although the "bidomain" one is far more complex, both models are reaction-diffusion systems [24, 3] of the general form

$$
\partial_{t} w=A w+F(w)
$$

where $A w=\nabla \cdot(\sigma(x) \nabla w)$ and $\sigma(x)$ is a positive symmetric matrix, eventually with $\operatorname{Ker} \sigma \neq\{0\}$. Only the monodomain model is addressed here.

Any microscopic description of the cell membrane can be inserted into the monodomain equations, providing a large variety of macroscopic models, ranging from 2 to about 100 equations. Although the approach would be the same for complex ones, this paper only treats the case of two simplified 2 variables models, namely the well-known FitzHugh-Nagumo one [7] and the one from Aliev-Panfilov [18]. The latter is very well suited to the myocardial cell, and often used in practical computer models $[17,21,22]$.

Computer models of the heart based on these equations (mono or bidomain, 2 or more ionic currents) currently are very popular in numerical electrophysiology. Because there may be many different time scales in the reaction terms, the solutions exhibit sharp propagating wave-fronts. For this reasons, only the recent improvement of computing capabilities allow 3D computations to be achieved. Moreover, until very recently, they were restricted to differences methods on structured grids and simple geometries $[17,19,13]$. A few researchers recently started to study computations on 3D unstructured meshes, coupled to an explicit, semi-implicit or fully-implicit time-stepping method $[14,2]$. The analysis of a Galerkin semidiscrete space approximation was conducted by S. Sanfelici [20]. To our knowledge, there has been no attempt at studying the effects of the time-stepping method on the stability of the approximation. As a matter of fact the problem of stability in time of fully discretized approximations is as difficult as the problem for global stability for the continuous solution of reaction-diffusion systems.

The main issue of this paper is to study the theoretical stability criterion for the 
explicit and semi-implicit Euler methods; and to derive error estimates for the approximate solutions.

Our idea is based on the proof of existence of global solutions to reaction-diffusion systems as presented in [24] : solutions for $t \in[0, T)$ extend to any $t>0$ due to the existence of strictly contracting regions $\Sigma$ for the flow $F(w)$. It is known [24] that such regions are invariant sets for regular enough solutions of the system (1). Here, we prove in theorems 7,9 and 11 that under suitable assumptions on the time-step, the regions $\Sigma$ are still invariants sets for the discrete solution, proving as a consequence $L^{\infty}$ bounds on the discrete solution. The convergence is proved and error estimates established in theorem 13.

Among the numerical methods suited to 3D computations on unstructured meshes, we choose a finite volume method introduced and analyzed in [6], well suited to general unstructured meshes and especially to mesh refinement, needed here to capture sharp wave-fronts. Moreover, it provides a sort of maximum principle, that may not be achieved for most finite element formulations but is the key ingredient of our proof.

The next section details the mathematical model, and recall some needed results of existence and stability for solutions for reaction-diffusion systems, essentially based on $[24,3,10]$. Section 3 briefly explains the finite volume technique for space discretization, and section 4 and 5 respectively concerns the stability and convergence results and proofs.

\section{The System of Partial Differential Equations}

\subsection{The Macroscopic Monodomain Model for Cardiac Electro-Cardiology}

At a microscopic scale, the surface membrane of the myocardial cells delimits an intra- and an extra-cellular medium, both containing ionic species. The model accounts for the dynamics of the trans-membrane ionic currents $I_{i o n}$ and difference of potential $u$, per surface unit. The membrane is considered to have a capacitive behaviour, so that the total current through the membrane is

$$
C \frac{d u}{d t}+I_{i o n}=I
$$

where $C$ is the capacitance per surface unit of the membrane. Furthermore, the cells are self-organized into myofibers in order to form the complete myocardium.

At a macroscopic scale, due to a homogenization process [8], the trans-membrane potential $u$ is defined on the whole heart $\Omega$ considered as the super-imposition 
of the intra- and extra-cellular medium. From the microstructure of the muscle fibers is derived at each point $x \in \Omega$ the positive definite tensor of conductivity $\sigma(x)=\operatorname{diag}\left(c_{l}, c_{t}, c_{t}\right)$ in the local orthonormal basis $\left(l, n_{1}, n_{2}\right)$, where $l$ is a unit vector tangent to the fiber at $x$. With the conductivity, the volumetric current can be expressed in terms of $u$, and equation (2) becomes

$$
\rho C \frac{d u}{d t}+\rho I_{i o n}=\nabla \cdot(\sigma \nabla u)
$$

where $\rho \gg 1$ is the ratio of membrane surface per unit of volume.

The fibers are tangent to the boundary $\partial \Omega$ of the heart. As a result the normal direction to the boundary at point $x \in \partial \Omega$ is an eigen-direction for $\sigma(x)$ and the conductivity tensor satisfies the following boundary condition :

$$
\forall x \in \partial \Omega, \quad \sigma(x) \cdot \mathbf{n}(x)=\lambda(x) \mathbf{n}(x) \quad(\lambda(x)>0),
$$

where $\mathbf{n}$ is the unit outward vector field on $\partial \Omega$.

First modeled by Hodgkin and Huxley in [11], the ionic current $I_{\text {ion }}$ decomposes into the contribution of several ionic channels $X_{i}$ :

$$
I_{i o n}=I_{X_{1}}+I_{X_{2}}+\ldots+I_{X_{p}}
$$

The states of the channels (open-closed) are described by gating variables $v=$ $\left(v_{1}, \ldots, v_{p}\right)$ which are controlled by ODEs,

$$
\frac{d v_{i}}{d t}=\varepsilon g_{i}\left(u, v_{i}\right)
$$

where the parameter $\varepsilon \ll 1$ means that the recovery variables have slow dynamics compared to the potential $u$. The ionic current through the channel $X_{i}$ depends on $u$ and $v$,

$$
I_{X_{i}}=-f_{i}(u, v) \text {. }
$$

Based on the original version, many such models have been constructed [1] according to moreless complex experimental studies of the cells membrane. Simplified versions of these models have been proposed, the simplest of which is the well known FitzHugh - Nagumo one [7, 16]. It writes

$$
I_{\text {ion }}=-f(u, v) \equiv u(u-1)(u-a)+v, \quad g(u, v)=k u-v,
$$

where $0<a<1$ and $k>0$ are given parameters. It will be referred to as the $F H N$ model. For, it is adapted from the original model of Hodkin-Huxley [11], it suits the behaviour of a nerve axon. For the myocardial cells, a simplified model was proposed by Aliev and Panfilov [18] and has been widely used in 3D simulations of the human ventricles $[17,21]$. It writes

$$
I_{i o n}=-f(u, v) \equiv k u(u-1)(u-a)+u v, \quad g(u, v)=k u(1+a-u)-v
$$


where $k>0$ and $0<a<1$ are still given parameters. It will be referred to as the AP model.

For sake of simplicity, only the case of the $A P$ and $F H N$ models are addressed, although the extension of our results to more complex ones shall be straightforward.

Equations (3), (5), (6), (7) rewrites in a dimensionless framework and for one gating variable $v$,

$$
\begin{aligned}
\varepsilon u_{t} & =\varepsilon^{2} \nabla \cdot(\sigma \nabla u)+f(u, v) \\
v_{t} & =g(u, v),
\end{aligned}
$$

where the functions $f, g: \mathbb{R}^{2} \mapsto \mathbb{R}$ are given by (8) for the $F H N$ model and by (9) for the $A P$ model.

The potential $u$ shall satisfy a Neumann boundary condition :

$$
\forall x \in \partial \Omega, \quad \sigma(x) \nabla u \cdot \mathbf{n}(x)=0,
$$

meaning that no current flows out of the heart. No additional boundary condition is needed concerning $v$, since it is ruled point wise by an ODE. Of course, an initial data is provided :

$$
\forall x \in \Omega, \quad u(x, 0)=u_{0}(x), v(x, 0)=v_{0}(x) .
$$

\subsection{Existence, Uniqueness and Regularity of Solutions}

General results for the Cauchy problem (10)-(13) are recalled here. Such systems of PDE have been widely studied [10, 24, 3]. Only basic non-exhaustive and nonoptimal results are recalled, that occur under reasonable assumptions expected from the physiological data. Furthermore, a framework for the proof of existence of solutions for all $t>0$ is drawn, that the numerical analysis will follow.

Theorem 1 (Local Existence and Uniqueness) The equations (10)-(13) are considered on a domain $\Omega \subset \mathbb{R}^{d}(d=1,2,3)$ with a $C^{2}$ regular boundary $\partial \Omega$. The conductivity tensor $\sigma$ is assumed $C^{1}$ regular on $\bar{\Omega}$ and such that

$$
\forall x \in \bar{\Omega}, \forall \xi \in \mathbb{R}^{d}, \quad \xi^{T} \sigma(x) \xi \geq 0 .
$$

The function $f$ and $g$ are assumed locally Lipschitz.

If the initial data satisfy $u_{0} \in H^{2}(\Omega)$, $u_{0}$ verifying the boundary condition (12); and $v_{0} \in L^{\infty}(\Omega)$, then the system (10)-(13) has a unique solution $w(x, t)=(u(x, t), v(x, t))$ on $\Omega \times[0, T)$ for some $T>0$, in the following (weak) sense : 
- the mapping $t \mapsto w(t) \in L^{2}(\Omega) \times L^{\infty}(\Omega)$ is continuous on $[0, T)$ with $w(0)=$ $\left(u_{0}, v_{0}\right)$,

- the mapping $t \mapsto w(t) \in L^{2}(\Omega) \times L^{\infty}(\Omega)$ is Frechet differentiable on $(0, T)$ with derivative $t \mapsto d w / d t(t) \in L^{2}(\Omega) \times L^{\infty}(\Omega)$,

- for $t \in(0, T)$, we have $u(\cdot, t) \in H^{2}(\Omega), f(w(\cdot, t)) \in L^{2}(\Omega)$ and $g(w(\cdot, t)) \in L^{\infty}(\Omega)$,

- for $t \in(0, T)$, equations (10), (11) and (12) respectively hold in $L^{2}(\Omega), L^{\infty}(\Omega)$ and $L^{2}(\partial \Omega)$.

- Moreover with the regularity assumed on the initial data, the mapping $t \mapsto w(t) \in$ $L^{\infty}(\Omega) \times L^{\infty}(\Omega)$ is continuous on $[0, T)$.

At last, note that $T=+\infty$ if the reaction terms $f, g$ are globally Lipschitz on $\mathbb{R}^{2}$.

Lemma 2 (Regularity) With the additional assumptions,

- the derivatives of $\sigma$ are $\mathrm{v}$-Hölder continuous on $\Omega$, for some $\mathrm{v}>0$ (ie $\sigma \in$ $C^{1+\mathrm{v}}(\Omega)$ ),

- $\sigma$ is uniformly elliptic on $\Omega$,

$$
\exists \alpha>0, \forall x \in \bar{\Omega}, \forall \xi \in \mathbb{R}^{d}, \quad \xi^{T} \sigma(x) \xi \geq \alpha|\xi|^{2} .
$$

- the initial data is such that $v_{0} \in C^{\mathrm{v}}(\Omega)$ for some $\mathrm{v}>0$,

the solution $w(x, t)$ is continuously differentiable in the variable t on $\bar{\Omega} \times(0, T)$ and $u(\cdot, t) \in C^{2}(\bar{\Omega})$ for $t \in(0, T)$. So, (10)-(13) hold in a classical (strong) sense.

\subsection{Stability of Solutions and Invariant Regions}

The solutions of theorem 1 exists only for $0<t<T$, where $T$ depends both on the initial data and on $f$ and $g$. But of course, only existence for all time $t>0$ makes sense in the physiological phenomena. For our solution to be relevant with the physiological framework it is moreover needed to have uniform $L^{\infty}$ bounds on $u$ and $v$. This is the main difficulty, referred to as stability. It can be studied in two ways.

First, assuming a polynomial growth at infinity for $f$ and $g$, Sobolev embeddings [15] are used to uniformly bound $u$ and $v$ in Sobolev spaces and then find solutions for all time $t \geq 0$, see $[10,25]$. Such techniques can be applied to solutions with weaker regularity as in lemma 2 . However $L^{\infty}$ bounds usually are unreachable although physiologically relevant.

The second way to study the stability is to construct invariant regions as developed in $[24,3]$. An invariant region for the Cauchy problem (10)-(13) is a closed subset $\Sigma \subset \mathbb{R}^{2}$ such that a solution of (10)-(13) having its initial data inside $\Sigma$ 's interior remains inside $\Sigma$. Such a solution is uniformly bounded in $L^{\infty}$ and moreover, since 
the restriction of $f$ and $g$ to $\Sigma$ are Lipschitz continuous, it has an infinite lifetime $T=+\infty$.

The second method is detailed here because it provides uniform $L^{\infty}$ bounds and is really perfectly suited to the numerical analysis below. It requires

- a good behaviour of the non-linear terms $f$ and $g$, so that invariant sets exist, see figure 1,

- a strong maximum principle for the operator $u \mapsto \nabla \cdot(\sigma \nabla u)$,

- regular solutions in order to apply the maximum principle.

Invariant regions for (10)-(13) are built by considering invariant regions of $\mathbb{R}^{2}$ for the reactive flow $(u, v) \in \mathbb{R}^{2} \mapsto(f(u, v), g(u, v)) \in \mathbb{R}^{2}$. For the heat equation $\partial_{t} u=$ $\nabla(\sigma \nabla u)$, intervals $\left[u_{-}, u_{+}\right]$are invariant regions. As a consequence, invariant sets $\Sigma$ are searched in the following form :

$$
\Sigma=\left\{(u, v) \in \mathbb{R}^{2}, u_{-} \leq u \leq u_{+}, v_{-} \leq v \leq v_{+}\right\}=\left[u_{-}, u_{+}\right] \times\left[v_{-}, v_{+}\right] .
$$

Definition 3 (Rectangular Invariant Set) The rectangular subset of $\mathbb{R}^{2}, \Sigma=\left[u_{-}, u_{+}\right] \times$ $\left[v_{-}, v_{+}\right]$is an invariant set for $f$ and $g$ if

$$
\forall(u, v) \in \Sigma, \quad \mid \begin{aligned}
& u=u_{-}, v_{-} \leq v \leq v_{+} \Rightarrow f(u, v)>0 \\
& u=u_{+}, v_{-} \leq v \leq v_{+} \Rightarrow f(u, v)<0 \\
& v=v_{-}, u_{-} \leq u \leq u_{+} \Rightarrow g(u, v)>0 \\
& v=v_{+}, u_{-} \leq u \leq u_{+} \Rightarrow g(u, v)<0 .
\end{aligned}
$$

For an invariant rectangular region $\Sigma$ (def. 3) to be invariant for (10)-(13), a strong point wise maximum principle is needed here. Remark that a simplification occurs in the scalar case (with one equation) where a Stampacchia-troncature technique can be used (see [4]).

Lemma 4 (Strong Maximum Principle) Let $\Omega$ be an open bounded subset of $\mathbb{R}^{d}$ whose boundary $\partial \Omega$ has $C^{2}$ regularity. Let $u \in C^{2}(\bar{\Omega})$ satisfy the boundary condition (12) for a tensor $\sigma \in C^{1}(\bar{\Omega})$ satisfying the boundary condition (4).

If $u$ has a maximum (resp. minimum) for $x \in \bar{\Omega}$ then $\nabla \cdot(\sigma \nabla u)(x) \leq 0$ (resp. $\nabla$. $(\sigma \nabla u)(x) \geq 0)$.

With lemma 4 invariant regions according to definition 3 are invariant regions for regular solutions of the PDE.

Theorem 5 (Invariant set for the PDE) Consider the system of equations (10)(13) with the assumptions of lemma 2. Moreover, assume that the conductivity tensor $\sigma$ verifies the boundary condition (4). 
If $\Sigma$ is a rectangular invariant set for $f$ and $g$, according to definition 3 , then it is an invariant region for (10)-(12) :

$$
\forall x \in \bar{\Omega}, w_{0}(x) \in \operatorname{int}(\Sigma) \Rightarrow \forall t>0, \forall x \in \bar{\Omega}, w(x, t) \in \Sigma
$$

and thus such a solution $w$ has an infinite lifetime $T=+\infty$.

Remark 6 For $\sigma=\lambda I d$, a proof has been given by J. Smoller in [24] when assuming that the boundary values of the solution $(u, v)_{\mid \partial \Omega}$, which are unknown here, remains inside $\Sigma$; and by A. Shcherbakov in [23] for a homogeneous Neumann boundary condition in the case of the FHN model (8). Lemma 4 and theorem 5 extend these results to the general case (10)-(12) for an anisotropic conductivity tensor satisfying (4).

Examples of invariant regions for the $F H N$ or $A P$ models (8), (9) are displayed on figure 1. Note that these invariant regions may be built as big as wishes, so that any regular solution of (10)-(12) remains uniformly bounded for all time $t \geq 0$.
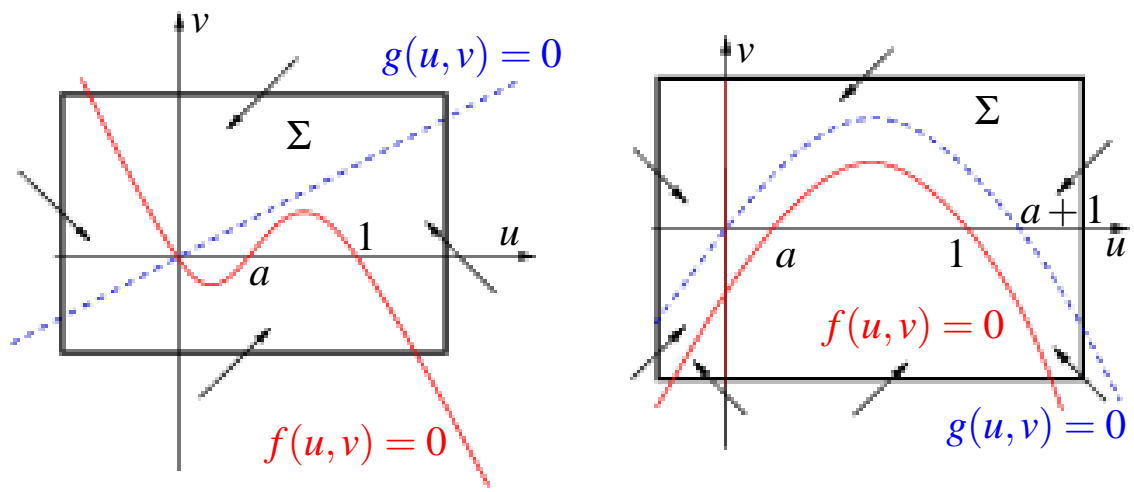

Fig. 1. Invariant regions $\Sigma$ for $F H N$ (left) and $A P$ (right) models

PROOF. [lemma 4.] At an interior point $x \in \Omega$ it is obvious. Assume that $u$ has a maximum for $x \in \partial \Omega$. With condition (4) one can construct an orthonormal basis $\mathcal{B}=\left(\xi_{1}, \ldots, \xi_{d}\right)$ such that $\sigma(x)=\operatorname{diag}\left(\lambda_{1}, \ldots, \lambda_{d}\right)$ in $\mathcal{B}\left(\right.$ with $\left.\lambda_{i} \geq 0, i=1, \ldots, d\right)$ and such that $\xi_{1}$ is normal to $\partial \Omega$ at $x$. Condition (12) together with (4) gives $\partial_{\xi_{1}} u(x)=0$. The family $\left(\xi_{2}, \ldots, \xi_{d}\right)$ generates the tangent hyper-surface of $\partial \Omega$ at point $x$. Since $u$ is $C^{2}(\partial \Omega)$ and its restriction to $\partial \Omega$ also has a local maximum at $x$, we have $\partial_{\xi_{i}} u(x)=0$ for $i=2, \ldots, d$. Consequently, $u \in C^{2}(\bar{\Omega})$ has a maximum in $x$ implies that $\partial_{\xi_{i}}^{2} u(x) \leq 0(i=1, \ldots, d)$. Now since $\sigma \in C^{1}(\bar{\Omega})$ one has $\nabla \cdot(\sigma \nabla u)(x)=\lambda_{1} \partial_{\xi_{1}}^{2} u(x)+\ldots+\lambda_{d} \partial_{\xi_{d}}^{2} u(x) \leq 0$.

PROOF. [theorem 5.] With the assumptions of lemma 2, let $w=(u, v)$ be a solution of (10)-(12) with initial value $w(0, \cdot)$ such that $w(0, x) \in \operatorname{int}(\Sigma)$ for all $x \in \bar{\Omega}$. 
We recall that $u$ is $C^{2}(\bar{\Omega})$ with respect to $x$ and that $w$ is $C^{1}$ with respect to $t$ on $\bar{\Omega} \times(0, T)$.

Imagine that $w(x, t)$ reaches the boundary $\partial \Sigma$ of $\Sigma$ at time $t_{0}$ and that $w(x, t) \in \Sigma$ for all $t \leq t_{0}$. Since $w(t):[0, T) \mapsto L^{\infty}(\Omega) \times L^{\infty}(\Omega)$ is continuous, $t_{0}>0$. Let $x_{0} \in \bar{\Omega}$ be such that $w\left(x_{0}, t_{0}\right) \in \partial \Sigma$.

We first assume that $w\left(x_{0}, t_{0}\right)$ is on the right side of $\partial \Sigma: u\left(x_{0}, t_{0}\right)=u_{+}$and $v_{-} \leq$ $v\left(x_{0}, t_{0}\right) \leq v_{+}$. On one hand, definition 3 implies that $f\left(w\left(x_{0}, t_{0}\right)\right)<0$; and on the other hand $u\left(\cdot, t_{0}\right)$ satisfies the conditions of the lemma 4 and $u\left(x_{0}, t_{0}\right)=\max _{\bar{\Omega}} u\left(\cdot, t_{0}\right)$. As a consequence, $\nabla \cdot(\sigma \nabla u)\left(x_{0}, t_{0}\right) \leq 0$. It proves that $\partial_{t} u\left(x_{0}, t_{0}\right)<0$. The function $\partial_{t} u$ being continuous on $\bar{\Omega} \times(0, T)$, there exists a neighbourhood $U$ of $\left(x_{0}, t_{0}\right)$ in $\bar{\Omega} \times\left(t_{0}, T\right)$ such that $\partial_{t} u<0$ on $U$, and therefore $u(x, t)<\max _{\bar{\Omega}} u\left(\cdot, t_{0}\right)=u_{+}$on $U$. Now imagine that $w(x, t)$ is on the top side of $\Sigma: v\left(x_{0}, t_{0}\right)=v_{+}$and $u_{-} \leq u\left(x_{0}, t_{0}\right) \leq$ $u_{+}$, then since $g<0$ on that top side, $\partial_{t} v\left(x_{0}, t_{0}\right)<0$ too and so there exists a neighbourhood $U$ of $\left(x_{0}, t_{0}\right)$ in $\bar{\Omega} \times\left(t_{0}, T\right)$ such that $v(x, t)<v_{+}$on $U$.

Altogether $w$ cannot get out of $\Sigma$ even at a corner point where the two precedent reasons both hold.

To end, $w$ remaining uniformly bounded, the reaction terms $f$ and $g$ can be considered as uniformly Lipschitz continuous and with the last remark of theorem $1 \mathrm{w}$ has an infinite lifetime $T=+\infty$.

\section{The Finite Volume Approximation}

\subsection{Meshes, Spaces and Notations}

We shall approximate the solutions of system (10)-(12) with a finite volume method according to the framework of [6], on admissible meshes adapted to the conductivity tensor $\sigma$. An admissible mesh of $\Omega$ (a bounded open subset of $\mathbb{R}^{d}$ whose boundary is piecewise $C^{1}$ ) adapted to $\sigma$ is given by :

(1) a set $\mathcal{T}$ of polygonal connected open subsets of $\Omega$, called cells and denoted by $K$, such that

$$
\bar{\Omega}=\cup_{K \in \mathcal{T}} \bar{K}, \quad \forall K, L \in \mathcal{T}, K \neq L \Rightarrow K \cap L=\emptyset .
$$

In the following $\mathrm{m}(K)$ will stand for the measure of a cell $K \in \mathcal{T}$. For a cell $K \in \mathcal{T}$ lying on the boundary, the edge $\bar{K} \cap \partial \Omega$ might be a $C^{1}$ curve, allowing non polygonal domains $\Sigma$. Two distinct cells $K$ and $L \in \mathcal{T}$ are called neighbour cells if $\bar{K} \cap \bar{L}$ has a non zero $(d-1)$-dimensional measure (i.e. non zero surface if $d=3$ or non zero length if $d=2$ ). On each cell $K \in \mathcal{T}$ a (positive definite) 
conductivity tensor $\sigma_{K} \in \mathbb{M}^{d \times d}$ is defined by

$$
\forall K \in \mathcal{T}, \quad \sigma_{K}=\frac{1}{\mathrm{~m}(K)} \int \sigma(x) d x .
$$

(2) A set $\mathcal{S}$ of interfaces, denoted by $e$ that are of two types :

- either there exists two neighbour cells $K, L \in \mathcal{T}$ such that $e=\bar{K} \cap \bar{L}, e$ is an internal interface and we set $e=K \mid L$;

- or there exists one cell $K \in \mathcal{T}$ such that $\bar{K} \cap \partial \Omega$ has a non zero $(d-1)$ dimensional measure and such that $e=\bar{K} \cap \partial \Omega, e$ is an external interface.

The set of internal interfaces is denoted by $S^{\star}$ and the set of external interfaces by $\delta \mathcal{S}$, and so $\mathcal{S}=\mathcal{S}^{\star} \cup \delta \mathcal{S}$. The $(d-1)$-dimensional measure for $e \in \mathcal{S}$ is $\mathrm{m}(e)$ and it is non zero. For $e \in \mathcal{S}$ and $K \in \mathcal{T}$ such that $e \subset \partial K$ we denote by $\mathbf{n}_{K, e}$ the unit vector normal to $e$ and pointing outward of $K$.

(3) Two sets of points $X=\left(x_{K}\right)_{K \in \mathcal{T}}, \mathcal{Y}=\left(y_{e}\right)_{e \in X}$, called cells and interfaces centers and such that $x_{K} \in K, y_{e} \in e$. We furthermore assume that for each cell $K \in \mathcal{T}$ and each interface $e \in \mathcal{S}$ such that $e \subset \partial K$,

$$
y_{e}-x_{K} \text { is co-linear to } \sigma_{K} \mathbf{n}_{K, e} \text {. }
$$

We denote by $d_{K, e}$ the euclidean distance $\left|y_{e}-x_{K}\right|$ and by $\lambda_{K, e}$ the (positive) proportionality coefficient between $\sigma_{K} \mathbf{n}_{K, e}$ and the unit vector $\left(y_{e}-x_{K}\right) / d_{K, e}$ :

$$
\sigma_{K} \mathbf{n}_{K, e}=\lambda_{K, e} \frac{y_{e}-x_{K}}{d_{K, e}}, \quad \text { and } \lambda_{K, e}>0
$$

Additionally, the boundary $\partial K$ of any cell $K \in \mathcal{T}$ can be spitted into internal and external interfaces, and we denote by $\delta K, \delta K^{\star}$, the subsets of $\mathcal{S}$ such that

$$
\bigcup_{e \in \delta K} e=\partial K, \quad \bigcup_{e \in \delta K^{\star}} e=\partial K \cap \Omega .
$$

We also define the size of the mesh as the maximum of the cells' diameters,

$$
\operatorname{size}(\mathcal{T})=\max _{K \in \mathcal{T}} \operatorname{diam}(K)
$$

As a consequence, a mesh is described by the collection $(\mathcal{T}, \mathcal{S}, \mathcal{X}, \mathcal{Y})$, but will be referred to as $\mathcal{T}$.

Examples of such meshes are given in [6]. In the isotropic case they are 2D meshes of triangles or 3D meshes of tetrahedra in which the centers $x_{K}$ are the centers of the circumscribed circles or spheres of the cells $K$, and more generally Voronoï meshes.

On an admissible mesh $\mathcal{T}$, the finite volume approximation for the solution of (10)(11) is a couple of functions $w_{T}=\left(u_{\mathcal{T}}, v_{\mathcal{T}}\right)$ piecewise constant on the cells $K \in \mathcal{T}$. 
As a consequence, we define

$$
L^{2}(\mathcal{T})=\left\{u_{\mathcal{T}}=\sum_{K \in \mathcal{T}} u_{K} \chi_{K},\left(u_{K}\right)_{K \in \mathcal{T}} \in \mathbb{R}^{N_{\mathcal{T}}}\right\} \subset L^{2}(\Omega),
$$

where $N_{\mathcal{T}}$ is the cardinal of $\mathcal{T}$, and $\chi_{K}(x)=1$ for $x$ in $K$ and 0 elsewhere. The space $L^{2}(\mathcal{T})$ is naturally handled with the inner product induced by $L^{2}(\Omega)$ and the associated norm :

$$
\left(u_{\mathcal{T}}, v_{\mathcal{T}}\right)_{L^{2}}=\sum_{K \in \mathcal{T}} u_{K} v_{K} \mathrm{~m}(K), \quad\left\|u_{\mathcal{T}}\right\|_{L^{2}}^{2}=\sum_{K \in \mathcal{T}}\left|u_{K}\right|^{2} \mathrm{~m}(K)
$$

This euclidean structure is extended to $L^{2}(\mathcal{T}) \times L^{2}(\mathcal{T})$. For $w=(u, v)$ and $\hat{w}=$ $(\hat{u}, \hat{v})$ we have

$$
(w, \hat{w})_{L^{2}}=(u, \hat{u})_{L^{2}}+(v, \hat{v})_{L^{2}}, \quad\|w\|_{L^{2}}^{2}=\|u\|_{L^{2}}^{2}+\|v\|_{L^{2}}^{2} .
$$

\subsection{Space Discretization}

In order to construct the finite volume approximation of system (10)-(11), the balance equation is written on any cell $K$ :

$$
\begin{aligned}
\varepsilon \frac{d}{d t} \int_{K} u d x & =\varepsilon^{2} \int_{\partial K} \sigma \nabla u \cdot \mathbf{n}_{K} d s+\int_{K} f(u, v) d x \\
\frac{d}{d t} \int_{K} v d x & =\int_{K} g(u, v) d x .
\end{aligned}
$$

Suppose that each value $u_{K}, v_{K}$ of the discrete solution approximates the mean value on $K$ of the exact solution $(u, v)$, then the discrete solution shall satisfy the following semi-discrete equation :

$$
\begin{aligned}
\varepsilon \frac{d u_{K}}{d t}(t) & =\frac{\varepsilon^{2}}{\mathrm{~m}(K)} \sum_{e \in \delta K} \phi_{K, e}\left(u_{\mathcal{T}}\right) \mathrm{m}(e)+f_{K}\left(u_{\mathcal{T}}, v_{\mathcal{T}}\right) \\
\frac{d v_{K}}{d t}(t) & =g_{K}\left(u_{\mathcal{T}}, v_{\mathcal{T}}\right)
\end{aligned}
$$

The terms $f_{K}\left(u_{\mathcal{T}}, v_{\mathcal{T}}\right)$ and $g_{K}\left(u_{\mathcal{T}}, v_{\mathcal{T}}\right)$ shall approximate $\frac{1}{\mathrm{~m}(K)} \int_{K} f(u, v) d x$ and $\frac{1}{\mathrm{~m}(K)} \int_{K} g(u, v) d x$ and are taken as follows :

$$
f_{K}\left(u_{\mathcal{T}}, v_{\mathcal{T}}\right)=f\left(u_{K}, v_{K}\right), \quad g_{K}\left(u_{\mathcal{T}}, v_{\mathcal{T}}\right)=g\left(u_{K}, v_{K}\right) .
$$

The term $\phi_{K, e}\left(u_{\mathcal{T}}\right)$ approximates the mean flux along $e \in S$ outward of $K$, specifically $\frac{1}{\mathrm{~m}(e)} \int_{e}(\sigma \nabla u) \cdot \mathbf{n}_{K} d s$. On the external interfaces the boundary condition (12) on 
$u$ is taken into account by fixing $\phi_{K, e}=0$. On the internal interfaces we approximate the flux as follows :

$$
\frac{1}{\mathrm{~m}(e)} \int_{e}(\sigma \nabla u) \cdot \mathbf{n}_{K} d s \simeq \nabla u\left(y_{e}\right) \cdot\left(\sigma_{K} \mathbf{n}_{K, e}\right)=\lambda_{K, e} \nabla u\left(y_{e}\right) \cdot \frac{y_{e}-x_{K}}{d_{K, e}} .
$$

An approximation of the derivative $\nabla u\left(y_{e}\right) \cdot \frac{y_{e}-x_{K}}{d_{K, e}}$ of $u$ at point $y_{e}$ is established by adding auxiliary unknowns $\left(u_{e}\right)_{e \in \mathcal{S}}$ at each point $\left(y_{e}\right)_{e \in \mathcal{S}}$ :

$$
\nabla u\left(y_{e}\right) \cdot \frac{y_{e}-x_{K}}{d_{K, e}} \simeq \frac{u_{e}-u_{K}}{d_{K, e}} .
$$

An additional requirement is that the numerical fluxes satisfy the conservativity property,

$$
\forall e=K \mid L \in \mathcal{S}^{\star}, \quad \phi_{K, e}=-\phi_{L, e} .
$$

This property enables us to determine the additional unknowns $u_{e}$ and to compute the numerical fluxes on the internal interfaces :

$$
\forall e=K \mid L \in \mathcal{S}^{\star}, \quad \phi_{K, e}=\tau_{e}\left(u_{L}-u_{K}\right)
$$

where

$$
\tau_{e}=\frac{\lambda_{K, e} \lambda_{L, e}}{\lambda_{K, e} d_{L, e}+\lambda_{L, e} d_{K, e}} \mathrm{~m}(e)>0 .
$$

The resulting approximation of the fluxes is consistent, as shown in [6].

consequently, the semi-discrete finite volume formulation is :

$$
\begin{aligned}
\varepsilon \frac{d u_{K}}{d t}(t) & =\frac{\varepsilon^{2}}{\mathrm{~m}(K)} \sum_{e=K \mid L \in \delta K^{\star}} \tau_{e}\left(u_{L}-u_{K}\right)+f\left(u_{K}, v_{K}\right), \\
\frac{d v_{K}}{d t}(t) & =g\left(u_{K}, v_{K}\right) .
\end{aligned}
$$

We recall that in (30)-(31) the boundary condition (12) is taken into account by fixing $\phi_{K, e}=0$ on the external interfaces.

The most natural initial data is given for all $K \in \mathcal{T}$ by $w_{K}(0)=w_{0}\left(x_{K}\right)$, or $w_{K}(0)=$ $\frac{1}{\mathrm{~m}(K)} \int_{K} w_{0}(x) d x$.

The discrete operator $A_{\mathcal{T}}$ defined on $L^{2}(\mathcal{T})$ by

$$
A_{\mathcal{T}}: u_{\mathcal{T}} \in L^{2}(\mathcal{T}) \mapsto z_{\mathcal{T}} \in L^{2}(\mathcal{T}), \quad z_{K}=\frac{1}{\mathrm{~m}(K)} \sum_{e=K \mid L \in \delta K^{\star}} \tau_{e}\left(u_{L}-u_{K}\right)
$$

approximates the continuous elliptic operator $u \mapsto \nabla \cdot(\sigma \nabla u)$.

At last, the semi-discrete system of ODEs simply writes 


$$
\begin{aligned}
\varepsilon \frac{d u_{\mathcal{T}}}{d t}(t) & =\varepsilon^{2} A_{\mathcal{T}} u_{\mathcal{T}}+f\left(u_{\mathcal{T}}, v_{\mathcal{T}}\right) \\
\frac{d v_{\mathcal{T}}}{d t}(t) & =g\left(u_{\mathcal{T}}, v_{\mathcal{T}}\right)
\end{aligned}
$$

The operator $A_{\mathcal{T}}$ is symmetric on $L^{2}(\mathcal{T})$ and verifies :

$$
\left(A_{\mathcal{T}} u_{\mathcal{T}}, u_{\mathcal{T}}\right)_{L^{2}(\Omega)}=-\sum_{e=K \mid L \in \mathcal{S}^{\star}} \tau_{e}\left|u_{L}-u_{K}\right|^{2} .
$$

Therefore $A_{\mathcal{T}}$ is non-negative and its kernel is the subspace of the constant functions on $\Omega$, and define the following semi-norm on $L^{2}(\mathcal{T})$,

$$
\left|u_{\mathcal{T}}\right|_{1, \mathcal{T}}^{2}=-\left(A_{\mathcal{T}} u_{\mathcal{T}}, u_{\mathcal{T}}\right)_{L^{2}}=\sum_{e=K \mid L \in \mathcal{S}^{\star}} \tau_{e}\left|u_{L}-u_{K}\right|^{2} .
$$

With this semi-norm the space of the finite volume functions will be referred to as $H^{1}(\mathcal{T})$. Unlike in the case of a finite element Galerkin formulation, the space $H^{1}(\mathcal{T})$ is not a subspace of $H^{1}(\Omega)$ but only a discrete equivalent.

\subsection{Time-Stepping Methods}

Given an admissible finite volume mesh as defined in section 3.1, we choose a time step $\Delta t>0$ and consider the forward Euler method (37)-(38) and the backward Euler method (39)-(40).

$$
\begin{aligned}
\varepsilon \frac{u_{\mathcal{T}}^{n+1}-u_{\mathcal{T}}^{n}}{\Delta t} & =\varepsilon^{2} A_{\mathcal{T}} u_{\mathcal{T}}^{n}+f\left(u_{\mathcal{T}}^{n}, v_{\mathcal{T}}^{n}\right), \\
\frac{v_{\mathcal{T}}^{n+1}-v_{\mathcal{T}}^{n}}{\Delta t} & =g\left(u_{\mathcal{T}}^{n}, v_{\mathcal{T}}^{n}\right) . \\
\varepsilon \frac{u_{\mathcal{T}}^{n+1}-u_{\mathcal{T}}^{n}}{\Delta t} & =\varepsilon^{2} A_{\mathcal{T}} u_{\mathcal{T}}^{n+1}+f\left(u_{\mathcal{T}}^{n}, v_{\mathcal{T}}^{n}\right), \\
& \frac{v_{\mathcal{T}}^{n+1}-v_{\mathcal{T}}^{n}}{\Delta t}=g\left(u_{\mathcal{T}}^{n}, v_{\mathcal{T}}^{n}\right) .
\end{aligned}
$$

\section{Stability Analysis}

As explained in section 2.3, any regular solution initially in a contracting rectangle $\Sigma$ (def. 3) exists for all time $t \geq 0$ and remains trapped in $\Sigma$. We shall prove in this 
section that

(1) the semi-discrete solutions of the ODEs (33)-(34) initially in $\Sigma$ exist for all $t>0$ and remain trapped in $\Sigma$ as well, without any additional regularity assumption on the mesh;

(2) the discrete solutions given by (37)-(38) or (39)-(40) initially in $\Sigma$ are welldefined for all $n \geq 0$ and remain trapped in $\Sigma$ as well, under classical conditions on the time step $\Delta t$.

Item (1) justifies the choice of a finite volume method, and proves that numerical instability are only caused by the time-stepping method. The $\Delta t$ conditions in item (2) splits into constraints due to the discrete elliptic operator $A_{\mathcal{T}}$ and the non-linear source terms $f_{K}, g_{K}$.

The balance between these constraints is ruled by the ratio of the mesh size $\operatorname{size}(\mathcal{T})$ to the time-scale factor $\varepsilon$, showing up the main question of the discretization : how should the mesh and the time step be chosen with respect to the value of $\varepsilon$ and the desired accuracy?

We recall that invariant regions can be built as big as one wishes (see figure 1) so that any solution of (10)-(12) associated with a bounded initial data can be approximated with numerical stability.

\subsection{Stability for the Semi-Discrete Problem}

Given any initial data $w_{\mathcal{T}}^{0} \in L^{2}(\mathcal{T}) \times L^{2}(\mathcal{T})$, the system of ODEs (33)-(34) has a unique solution $w \in \mathrm{C}^{1}\left([0, T) ; L^{2}(\mathcal{T}) \times L^{2}(\mathcal{T})\right)$, for some $T>0$, because $f$ and $g$ are locally Lipschitz on $\mathbb{R}^{2}$.

Theorem 7 Let $\Sigma \subset \mathbb{R}^{2}$ be a rectangular invariant set (def. 3). Then $\Sigma$ is an invariant region for the semi discrete system (33)-(34) :

$$
\forall K \in \mathcal{T}, w_{K}^{0} \in \Sigma \Rightarrow \forall t>0, \forall K \in \mathcal{T}, w_{K}(t) \in \Sigma .
$$

and $w$ has an infinite lifetime $T=+\infty$

The proof of the theorem is supported by the following lemma which is a discrete analogue of lemma 4.

Lemma 8 Let $\mathcal{T}$ be an admissible finite volume mesh of $\Omega$ adapted to the conductivity tensor $\sigma$ and $A_{\mathcal{T}}$ be the operator defined by (32).

If $u_{\mathcal{T}}$ has a maximum (resp. minimum) for $K \in \mathcal{T}$ then $\left\{A_{\mathcal{T}} u_{\mathcal{T}}\right\}_{K} \leq 0$ (resp. $\left\{A_{\mathcal{T}} u_{\mathcal{T}}\right\}_{K} \geq$ $0)$. 
PROOF. [Lemma 8] If $u_{\mathcal{T}} \in L^{2}(\mathcal{T})$ has a maximum for $K \in \mathcal{T}$ then for any cell $L \in \mathcal{T}$ neighbouring $K$ one has $u_{K} \geq u_{L}$. As a result $u_{L}-u_{K}$ is non-positive and so $\left\{A_{\mathcal{T}} u_{\mathcal{T}}\right\}_{K} \leq 0$

PROOF. [Theorem 7] Let $\Sigma$ be an invariant rectangle and $w^{0} \in L^{2}(\mathcal{T}) \times L^{2}(\mathcal{T})$ satisfy $w_{K}^{0} \in \Sigma$ for all $K \in \mathcal{T}$. Consider $T>0$ and the solution $w \in C^{1}\left([0, T] ; L^{2}(\mathcal{T}) \times\right.$ $\left.L^{2}(\mathcal{T})\right)$ of (33)-(34) with initial data $w_{\mathcal{T}}^{0}$.

Assume now that $w$ reaches $\partial \Sigma$ at time $t_{0} \geq 0$ and that $w_{K}(t) \in \Sigma$ for all $K \in \mathcal{T}$ and all $t \in\left[0, t_{0}\right]$. Let $K \in \mathcal{T}$ be such that $w_{K}\left(t_{0}\right) \in \partial \Sigma$.

First assume that $w_{K}\left(t_{0}\right)$ is on the right side of $\partial \Sigma: u_{K}\left(t_{0}\right)=u_{+}$and $v_{-} \leq v_{K}\left(t_{0}\right) \leq$ $v_{+}$. Then, on the one hand definition 3 implies $f\left(w_{K}\left(t_{0}\right)\right)<0$, and on the other hand $\max _{L \in \mathcal{T}} u_{L}\left(t_{0}\right)=u_{+}=u_{K}\left(t_{0}\right)$ so that property $\left\{A_{\mathcal{T}} u_{\mathcal{T}}\left(t_{0}\right)\right\}_{K} \leq 0$ (lemma 8). As a result we have $d u_{K} / d t\left(t_{0}\right)<0$ and so $u_{K}(t)<u_{+}$for $t \in\left(t_{0}, t_{0}+\delta\right)$ for some $\delta>0$.

Now if $w_{K}\left(t_{0}\right)$ is on the top side of $\Sigma, v_{K}\left(t_{0}\right)=v_{+}$and $u_{-} \leq u_{K}\left(t_{0}\right) \leq u_{+}$, since $g<0$ on that top side then $\partial_{t} v_{K}\left(t_{0}\right)<0$ and so $v_{K}(t)<v_{+}$for $t \in\left(t_{0}, t_{0}+\delta\right)$.

$\mathrm{Al}$ together, $w$ cannot get out of $\Sigma$, even at a corner point where the two precedent reasons hold.

To end, since $w$ remain uniformly bounded it has an infinite lifetime $T=+\infty$.

\subsection{Stability for the Semi-Implicit Euler Method}

We recall that the operator $A_{\mathcal{T}}$ is non-positive, so that $\mathrm{Id}-\varepsilon \Delta t A_{\mathcal{T}}$ is symmetric and positive-definite for any $\Delta t>0$. As a consequence, given $\left(u_{\mathcal{T}}^{n}, v_{\mathcal{T}}^{n}\right)$, equation (39) has a unique solution; and for any $w_{\mathcal{T}}^{0}$, equations (39)-(40) define a unique sequence $\left(w_{\mathcal{T}}^{n}\right)_{n \in \mathbb{N}}$ in $L^{2}(\mathcal{T}) \times L^{2}(\mathcal{T})$.

The following lemma gives a condition on $\Delta t$ for $w_{\mathcal{T}}^{n}$ to remain in $\Sigma$ if $w_{\mathcal{T}}^{0} \in \Sigma$.

Theorem 9 Let $\Sigma$ be a rectangular invariant set (def 3). If the time step $\Delta t$ verifies

$$
\frac{\Delta t}{\varepsilon}\left|\min _{\Sigma} \partial_{u} f\right| \leq 1, \quad \Delta t\left|\min _{\Sigma} \partial_{v} g\right| \leq 1,
$$

then $\Sigma$ is an invariant region for the solution $\left(w_{\mathcal{T}}^{n}\right)_{n \in \mathbb{N}}$ of (39)-(40) :

$$
\forall K \in \mathcal{T}, w_{K}^{0} \in \Sigma \Rightarrow \forall n \in \mathbb{N}, \forall K \in \mathcal{T}, w_{K}^{n} \in \Sigma
$$

Remark 10 Condition (41) can be specified with $F(u)=-u(u-a)(u-1)$ : 
- for the FHN model (8), we have

$$
\left|\min _{\Sigma} \partial_{u} f\right|=\max \left(\left|F^{\prime}\left(u_{-}\right)\right|,\left|F^{\prime}\left(u_{+}\right)\right|\right), \quad\left|\min _{\Sigma} \partial_{v} g\right|=1,
$$

- for the AP model (9), we have

$$
\left|\min _{\Sigma} \partial_{u} f\right|=\max \left(\left|F^{\prime}\left(u_{-}\right)-v_{+}\right|,\left|F^{\prime}\left(u_{+}\right)-v_{+}\right|\right), \quad\left|\min _{\Sigma} \partial_{v} g\right|=1 .
$$

This yields explicit computations of the time-step in applied cases.

PROOF. [Lemma 9] Equations (39)-(40) can be rewritten as

$$
\begin{aligned}
\left(\mathrm{Id}-\varepsilon \Delta t A_{\mathcal{T}}\right) u_{\mathcal{T}}^{n+1} & =u_{\mathcal{T}}^{n}+\Delta t f\left(w_{\mathcal{T}}^{n}\right) / \varepsilon, \\
v_{\mathcal{T}}^{n+1} & =v_{\mathcal{T}}^{n}+\Delta t g\left(w_{\mathcal{T}}^{n}\right),
\end{aligned}
$$

for all $n \in \mathbb{N}$, which has a unique solution (see above).

Let us consider the following function defined on $\mathbb{R}^{2}$ :

$$
\phi(w)=\left(\phi_{1}(w), \phi_{2}(w)\right)=(u+\Delta t f(w) / \varepsilon, v+\Delta t g(w)) .
$$

Under condition (41) one has $\partial_{u} \phi_{1} \geq 0$ and $\operatorname{sos} \sup _{\Sigma} \phi_{1}=\phi_{1}\left(u_{+}, v\right)=u_{+}+\Delta t f\left(u_{+}, v\right) / \varepsilon$ for some $v, v_{-} \leq v \leq v_{+}$. But definition 3 ensures that $f\left(u_{+}, v\right)<0$ and then $\sup _{\Sigma} \phi_{1} \leq u_{+}$. Similarly, $\inf _{\Sigma} \phi_{1} \geq u_{-}$and $v_{-} \leq \inf _{\Sigma} \phi_{2} \leq \sup _{\Sigma} \phi_{2} \leq v_{+}$. As a consequence, $\phi(\Sigma) \subset \Sigma$.

Now let $w_{\mathcal{T}}^{0} \in L^{2}(\mathcal{T}) \times L^{2}(\mathcal{T})$ satisfy $w_{K}^{0} \in \Sigma$ for all $K \in \mathcal{T}$. Since $\phi(\Sigma) \subset \Sigma$ we have $\left(\left\{\left(\operatorname{Id}-\varepsilon \Delta t A_{\mathcal{T}}\right) u_{\mathcal{T}}^{1}\right\}_{K}, v_{K}^{1}\right) \in \Sigma$ for all $K \in \mathcal{T}$. If $K \in \mathcal{T}$ is such that $u_{K}^{1}=$ $\max _{L \in \mathcal{T}} u_{L}^{1}$, then $\left\{A_{\mathcal{T}} u_{\mathcal{T}}^{1}\right\}_{K} \leq 0$ (this is lemma 8) and then $\left\{\left(\operatorname{Id}-\varepsilon \Delta t A_{\mathcal{T}}\right) u_{\mathcal{T}}^{1}\right\}_{K} \leq$ $u_{+}$implies that $u_{K}^{1}=\max _{L \in \mathcal{T}} u_{L}^{1} \leq u_{+}$. Similarly, $\inf _{L \in \mathcal{T}} u_{L}^{1} \geq u_{-}$and so $w_{K}^{1} \in \Sigma$ for all $K \in \mathcal{T}$

\subsection{Stability for the Explicit Euler Method}

Given any $w_{\mathcal{T}}^{0} \in L^{2}(\mathcal{T}) \times L^{2}(\mathcal{T})$, the discrete system (37)-(38) define explicitly a unique sequence $\left(w_{\mathcal{T}}^{n}\right)_{n \in \mathbb{N}}$ in $L^{2}(\mathcal{T}) \times L^{2}(\mathcal{T})$.

The following lemma gives a condition on $\Delta t$ for $w_{\mathcal{T}}^{n}$ to remain in $\Sigma$ if $w_{\mathcal{T}}^{0} \in \Sigma$.

Theorem 11 Let $\Sigma$ be a rectangular invariant set (def 3). If the time step $\Delta t$ verifies

$$
\forall K \in \mathcal{T}, \quad \Delta t \frac{\varepsilon}{\mathrm{m}(K)} \sum_{e \in \delta K^{\star}} \tau_{e}+\frac{\Delta t}{\varepsilon}\left|\inf _{\Sigma} \partial_{u} f\right| \leq 1, \quad \Delta t\left|\min _{\Sigma} \partial_{v} g\right| \leq 1,
$$


then $\Sigma$ is an invariant region for the solution $\left(w_{\mathcal{T}}^{n}\right)_{n \in \mathbb{N}}$ of (37)-(38):

$$
\forall K \in \mathcal{T}, w_{K}^{0} \in \Sigma \Rightarrow \forall n \in \mathbb{N}, \forall K \in \mathcal{T}, w_{K}^{n} \in \Sigma
$$

Remark 12 There is a classical condition of regularity for a family of admissible meshes that is there exist uniform constants $\alpha, \beta>0$ such that

$$
\forall e=K \mid L \in \mathcal{S}^{\star}, \quad \alpha \Delta x \leq d_{K, e}+d_{L, e}, \quad \forall K \in \mathcal{T}, \quad \Delta x \mathrm{~m}(\partial K) \leq \beta \mathrm{m}(K),
$$

where $\Delta x$ is the size of the mesh $\mathcal{T}$. For such a family of admissible meshes, and in the isotropic case $\nabla \cdot(\sigma \nabla u)=D \Delta(u)$, the first stability condition (42) becomes

$$
\varepsilon D \frac{\Delta t}{\Delta x^{2}} \frac{\beta}{\alpha}+\frac{\Delta t}{\varepsilon}\left|\inf _{\Sigma} \partial_{u} f\right| \leq 1
$$

This condition combines the classical stability conditions for both the heat equation $u^{\prime}=D \Delta(u)$ and the ordinary differential equation $u^{\prime}=f(u)$.

PROOF. [Lemma 11] Equations (37)-(38) can be rewritten as :

$$
\begin{aligned}
& u_{\mathcal{T}}^{n+1}=\left(\operatorname{Id}+\varepsilon \Delta t A_{\mathcal{T}}\right) u_{\mathcal{T}}^{n}+\frac{\Delta t}{\varepsilon} f\left(w_{\mathcal{T}}^{n}\right), \\
& v_{\mathcal{T}}^{n+1}=v_{\mathcal{T}}^{n}+\Delta t g\left(w_{\mathcal{T}}^{n}\right) .
\end{aligned}
$$

Let $w_{\mathcal{T}}^{0} \in L^{2}(\mathcal{T}) \times L^{2}(\mathcal{T})$ satisfy $w_{K}^{0} \in \Sigma$ for all $K \in \mathcal{T}$. For any $K \in \mathcal{T}$,

$$
\phi^{-}\left(w_{K}^{0}\right) \leq u_{K}^{1} \leq \phi^{+}\left(w_{K}^{0}\right)
$$

where the two functions $\phi^{-}$and $\phi^{+}$are defined by

$$
\begin{aligned}
& \phi^{-}(w)=u+\frac{\varepsilon \Delta t}{\mathrm{~m}(K)} \sum_{e \in \delta K^{\star}} \tau_{e}\left(u_{-}-u\right)+\frac{\Delta t}{\varepsilon} f(w), \\
& \phi^{+}(w)=u+\frac{\varepsilon \Delta t}{\mathrm{~m}(K)} \sum_{e \in \delta K^{\star}} \tau_{e}\left(u_{+}-u\right)+\frac{\Delta t}{\varepsilon} f(w) .
\end{aligned}
$$

The stability condition (42) implies that $\partial_{u} \phi^{-} \geq 0$ and $\partial_{u} \phi^{+} \geq 0$ on $\Sigma$, and then,

$$
u_{-}+\Delta t f\left(u_{-}, v_{K}^{0}\right) \leq u_{K}^{1} \leq u_{+}+\Delta t f\left(u_{+}, v_{K}^{0}\right)
$$

At last, $\Sigma$ being an invariant rectangle (def. 3), $f\left(u_{-}, v_{K}^{0}\right)>0$ and $f\left(u_{+}, v_{K}^{0}\right)<0$. As a consequence, $u_{-} \leq u_{K}^{1} \leq u_{+}$. Similarly we have $v_{-} \leq v_{K}^{1} \leq v_{+}$and at last, $w_{K}^{1} \in \Sigma$ for all $K \in \mathcal{T}$ 


\section{Convergence Analysis}

Convergence of the finite volume approximations and error estimates are proved in this section.

The functions $f, g$ are supposed to be those of the $F H N$ or $A P$ model, and the other data $\Omega, \sigma, w^{0}=\left(u^{0}, v^{0}\right)$ are supposed to fulfill the assumptions of lemma 2 and theorem 5, in order for the solution $w(t)$ to exists for all $t>0$ in a fixed rectangle $\Sigma$, depending only on $w^{0}$.

In this case, the solution $w(x, t)$ is $C^{2}(\bar{\Omega})$ with respect to $x$ and $C^{1}([0,+\infty))$ with respect to $t$.

Given an admissible finite volume mesh as defined in section 3.1, and $\Delta t>0$, we denote by $\left(\bar{w}_{\mathcal{T}}^{n}\right)_{n \in \mathbb{N}}$ the sequence defined by (39)-(40) or (37)-(38) and $\bar{w}_{K}^{0}=$ $w^{0}\left(x_{K}\right)$ for all $K \in \mathcal{T}$.

Under the condition (41) or (42), both $w$ and $\bar{w}_{\mathcal{T}}^{n}$ remain in $\Sigma$.

In order to compare the discrete and the continuous solutions we introduce the sequence $\left(w_{\mathcal{T}}^{n}\right)_{n \in \mathbb{N}}$ in $L^{2}(\mathcal{T}) \times L^{2}(\mathcal{T})$ defined by

$$
w_{K}^{n}=w\left(x_{K}, t^{n}\right)=\left(u\left(x_{K}, t^{n}\right), v\left(x_{K}, t^{n}\right)\right) .
$$

The error $\left(e_{\mathcal{T}}^{n}\right)_{n \in \mathbb{N}}$ writes

$$
e_{\mathcal{T}}^{n}=w_{\mathcal{T}}^{n}-\bar{w}_{\mathcal{T}}^{n} \in L^{2}(\mathcal{T}) \times L^{2}(\mathcal{T})
$$

Theorem 13 (Convergence and Error Estimate) Suppose that the data fulfill the assumptions of lemma 2 and theorem 5. Assume furthermore that $\Sigma \subset \mathbb{R}^{2}$ is an invariant rectangle (def. 3) for $f$ and $g$ such that the initial data $w^{0}$ is in $\Sigma$ 's interior.

We additionally assume that $\partial_{t} w$ and the second order derivatives in space $\partial_{\xi_{i}}^{2} u$ of $u$ are uniformly bounded on $\bar{\Omega} \times(0, T]$.

Let $w_{\mathcal{T}}^{n}$ be the approximation of $w$ as defined by (37)-(38) (or in (39)-(40)) with the initial data

$$
\forall K \in \mathcal{T}, \quad \bar{w}_{K}^{0}=w_{0}\left(x_{K}\right)=\left(u_{0}\left(x_{K}\right), v_{0}\left(x_{K}\right)\right) .
$$

If the stability condition (41) (or (42)) relative to $\Sigma$ is satisfied, then there exists two constants $C$ and $\mu$, only depending on the data $\left(\Omega, w^{0}, f, g\right.$ and $\left.\Sigma\right)$ such that for $n \Delta t \leq T$ the error is

$$
\left\|e_{\mathcal{T}}^{n}\right\|_{L^{2}} \leq C e^{\mu T}(\operatorname{size}(\mathcal{T})+\Delta t) .
$$

PROOF. [Theorem 13] We shall prove theorem 13 for the Euler semi implicit 
scheme (37)-(38), the proof being similar for the Euler semi explicit scheme (39)(40). For simplicity we shall also take $\varepsilon=1$. With the notations previously defined, the balance equation at time $t^{n+1}$ for (10)-(12) on any cell $K \in \mathcal{T}$ reads :

$$
\begin{aligned}
\frac{d}{d t} \int_{K} u\left(x, t^{n+1}\right) d x & \left.=\int_{\partial K \cap \Omega} \sigma \nabla u\left(x, t^{n+1}\right)\right) \cdot \mathbf{n}_{K} d s+\int_{K} f\left(w\left(x, t^{n+1}\right)\right) d x \\
\frac{d}{d t} \int_{K} v\left(x, t^{n+1}\right) d x & =\int_{K} g\left(w\left(x, t^{n+1}\right)\right) d x .
\end{aligned}
$$

together with definition (43) this leads to :

$$
\begin{aligned}
& \frac{u_{K}^{n+1}-u_{K}^{n}}{\Delta t}+T_{K}^{1, n}=\left\{A_{\mathcal{T}} u_{\mathcal{T}}^{n+1}\right\}_{K}+\frac{1}{\mathrm{~m}(K)} \sum_{e \in \delta K^{\star}} F_{e, K}^{n}+f\left(w_{K}^{n}\right)+R_{K}^{1, n} \\
& \frac{v_{K}^{n+1}-v_{K}^{n}}{\Delta t}+T_{K}^{2, n}=g\left(w_{K}^{n}\right)+R_{K}^{2, n},
\end{aligned}
$$

where :

- $F_{e, K}^{n}$ stands for the consistence error on the numerical approximation of the flux $\int_{e} \sigma \nabla u \cdot \mathbf{n}_{K, e}$ on the edge $e \in \delta K^{\star}$ :

$$
\int_{e} \sigma(x) \nabla u\left(x, t_{n+1}\right) \cdot \mathbf{n}_{K} d s=\tau_{e}\left(u_{L}^{n+1}-u_{K}^{n+1}\right)+F_{e, K}^{n} \mathrm{~m}(e),
$$

$F_{e K}^{n}$ fulfills the following conservativity property :

$$
\forall e=K \mid L \in \mathcal{S}^{\star}, \quad F_{e, K}^{n}=-F_{e, L}^{n},
$$

and since $u$ is assumed to have uniformly bounded second order derivatives on $\bar{\Omega} \times(0, T]$ it is controlled by the size of the mesh (see [6]) :

$$
\left|F_{e, K}^{n}\right| \leq C_{w, \sigma} \operatorname{size}(\mathcal{T})
$$

(where $C_{\alpha}$ generically denotes a constant depending on the data $\alpha$ only).

- $T_{K}^{n}=\left(T_{K}^{1, n}, T_{K}^{2, n}\right)$ stands for the consistence error on the time integration :

$$
\frac{1}{\mathrm{~m}(K)} \int_{K} \partial_{t} w\left(x, t_{n+1}\right) d x=\frac{w_{K}^{n+1}-w_{K}^{n}}{\Delta t}+T_{K}^{n}
$$

which is of order one since $\partial_{t} w$ is uniformly bounded on $\bar{\Omega} \times(0, T]$ :

$$
\left|T_{K}^{n}\right| \leq C_{w}(\operatorname{size}(\mathcal{T})+\Delta t)
$$

We shall consider $T_{T}^{n}$ as a finite volume function $T_{\mathcal{T}}^{n} \in L^{2}(\mathcal{T}) \times L^{2}(\mathcal{T})$. 
- $\quad R_{K}^{n}=\left(R_{K}^{1, n}, R_{K}^{2, n}\right)$ is the consistence error on the reaction term, for $F=(f, g)$ :

$$
\frac{1}{\mathrm{~m}(K)} \int_{K} F\left(w\left(x, t_{n+1}\right)\right) d x=F\left(w_{K}^{n+1}\right)+R_{K}^{n} .
$$

Since $w$ remains bounded (inside $\Sigma$ ) and $f, g$ are locally Lipschitz, it is of order one :

$$
\left|R_{K}^{n}\right| \leq C_{w, f, g, \Sigma} \operatorname{size}(\mathcal{T})
$$

Again we shall consider $R_{\mathcal{T}}^{n}$ as a finite volume function $R_{\mathcal{T}}^{n} \in L^{2}(\mathcal{T}) \times L^{2}(\mathcal{T})$. Now, subtracting (39)- (40) to (46)-(47), the error $e_{\mathcal{T}}^{n}$ defined in (44) satisfies the following equation :

$$
\begin{aligned}
& \frac{e_{K}^{1, n+1}-e_{K}^{1, n}}{\Delta t}+T_{K}^{1, n}=\left\{A_{\mathcal{T}} e_{\mathcal{T}}^{1, n+1}\right\}_{K}+\frac{1}{\mathrm{~m}(K)} \sum_{e \in \delta K^{\star}} F_{e, K}^{n} \mathrm{~m}(e)+\left(f\left(w_{K}^{n}\right)-f\left(\bar{w}_{K}^{n}\right)\right)+R_{K}^{1, n} \\
& \frac{e_{K}^{2, n+1}-e_{K}^{2, n}}{\Delta t}+T_{K}^{2, n}=g\left(w_{K}^{n}\right)-g\left(\bar{w}_{K}^{n}\right)+R_{K}^{2, n}
\end{aligned}
$$

multiplying the first equation by $\mathrm{m}(K) e_{K}^{1, n+1}$ and summing over all cells $K \in \mathcal{T}$ leads to, by making use of the inner product (20), of the discrete $H^{1}$ semi-norm (36) and of the formula (35) :

$$
\begin{aligned}
\frac{1}{\Delta t}\left(e_{\mathcal{T}}^{1, n+1}, e_{\mathcal{T}}^{1, n+1}-e_{\mathcal{T}}^{1, n}\right)_{L^{2}}+\left|e_{\mathcal{T}}^{1, n+1}\right|_{1, \mathcal{T}}^{2} & =\left(e_{\mathcal{T}}^{1, n+1}, R_{\mathcal{T}}^{1, n}-T_{\mathcal{T}}^{1, n}\right)_{L^{2}} \\
& +\left(e_{\mathcal{T}}^{1, n+1}, f\left(w_{\mathcal{T}}^{n}\right)-f\left(\bar{w}_{\mathcal{T}}^{n}\right)\right)_{L^{2}} \\
& +\sum_{K \in \mathcal{T}} e_{K}^{1, n+1} \sum_{e \in \delta K^{\star}} F_{e, K}^{n} \mathrm{~m}(e)
\end{aligned}
$$

First of all, since the restriction to $\Sigma$ of $f$ is Lipschitz continuous, there is a constant $\Lambda_{f, \Sigma}$ such that : $\left\|f\left(w_{\mathcal{T}}^{n}\right)-f\left(\bar{w}_{\mathcal{T}}^{n}\right)\right\|_{L^{2}} \leq \Lambda\left\|e_{\mathcal{T}}^{n}\right\|_{L^{2}}$. Then, by making use of the Schwartz inequality :

$$
\left|\left(e_{\mathcal{T}}^{1, n+1}, f\left(w_{\mathcal{T}}^{n}\right)-f\left(\bar{w}_{\mathcal{T}}^{n}\right)\right)_{L^{2}}\right| \leq \Lambda\left\|e_{\mathcal{T}}^{n}\right\|_{L^{2}}\left\|e_{\mathcal{T}}^{1, n+1}\right\|_{L^{2}}
$$

with the Schwartz inequality again :

$$
\begin{aligned}
\left|\left(e_{\mathcal{T}}^{1, n+1}, e_{\mathcal{T}}^{1, n}\right)_{L^{2}}\right| & \leq\left\|e_{\mathcal{T}}^{1, n+1}\right\|_{L^{2}}\left\|e_{\mathcal{T}}^{n}\right\|_{L^{2}}\left|\left(e_{\mathcal{T}}^{1, n+1}, R_{\mathcal{T}}^{1, n}-T_{\mathcal{T}}^{1, n}\right)\right| \\
& \leq \underbrace{\left(\left\|R_{\mathcal{T}}^{n}\right\|_{L^{2}}+\left\|T_{\mathcal{T}}^{n}\right\|_{L^{2}}\right)}_{\leq C_{w, \Omega, f, g, \Sigma}(\Delta t+\operatorname{size}(\mathcal{T}))}\left\|e_{\mathcal{T}}^{1, n+1}\right\|_{L^{2}} .
\end{aligned}
$$

The conservativity (48) of $F_{K, e}^{n}$ reads : 


$$
\begin{aligned}
\left|\sum_{K \in \mathcal{T}} e_{K}^{1, n+1} \sum_{e \in \delta K^{\star}} F_{e, K}^{n} \mathrm{~m}(e)\right| & =\left|\sum_{e=K \mid L \in \mathcal{S}^{\star}} F_{e, K}^{n}\left(e_{K}^{1, n+1}-e_{L}^{1, n+1}\right) \mathrm{m}(e)\right| \\
& \leq\left|e_{\mathcal{T}}^{1, n+1}\right|_{1, \mathcal{T}} \underbrace{\left(\sum_{e \in \mathcal{S}^{\star}}\left|F_{e}^{n}\right|^{2} \mathrm{~m}(e)^{2} / \tau_{e}\right)^{1 / 2}}_{\leq C_{w} \operatorname{size}(\mathcal{T}) \sum_{e \in \mathcal{S}^{\star}} \mathrm{m}(e)^{2} / \tau_{e}} .
\end{aligned}
$$

the conductivity tensor being uniformly elliptic on $\Omega, \sum_{e \in \mathcal{S}^{\star}} \mathrm{m}(e)^{2} / \tau_{e} \leq C_{\sigma} m(\Omega)$, where $m(\Omega)$ is the measure of the domain $\Omega$. Altogether with equation (53) these upper bounds lead to :

$$
\begin{aligned}
\frac{1}{\Delta t}\left\|e_{\mathcal{T}}^{1, n+1}\right\|_{L^{2}}^{2}+\left|e_{\mathcal{T}}^{1, n+1}\right|_{1, \mathcal{T}}^{2} & \leq\left(\Lambda+\frac{1}{\Delta t}\right)\left\|e_{\mathcal{T}}^{1, n+1}\right\|_{L^{2}}\left\|e_{\mathcal{T}}^{n}\right\|_{L^{2}} \\
& +C(\operatorname{size}(\mathcal{T})+\Delta t)\left(\left\|e_{\mathcal{T}}^{1, n+1}\right\|_{L^{2}}+\left|e_{\mathcal{T}}^{1, n+1}\right|_{1, \mathcal{T}}\right)
\end{aligned}
$$

and using Young's inequalities for the three terms on right hand side writes :

$$
\left\|e_{\mathcal{T}}^{1, n+1}\right\|_{L^{2}}^{2} \leq \frac{(1+\Lambda \Delta t)^{2}}{1-\Delta t}\left\|e_{\mathcal{T}}^{n}\right\|_{L^{2}}^{2}+C(\operatorname{size}(\mathcal{T})+\Delta t)^{2} \Delta t
$$

Using the same process on (52) gives the same upper bound on $\left\|e_{\mathcal{T}}^{2, n+1}\right\|_{L^{2}}^{2}$ and so, if $(n+1) \Delta t \leq T$ one has :

$$
\left\|e_{\mathcal{T}}^{n+1}\right\|_{L^{2}}^{2} \leq e^{\mu T}\left(\left\|e_{\mathcal{T}}^{0}\right\|_{L^{2}}^{2}+C(\operatorname{size}(\mathcal{T})+\Delta t)^{2}\right)
$$

for some constant $\mu$ related with $\Lambda$, which ends the proof for theorem 13 .

\section{References}

[1] Alain Bardou, Pierre Auger, Pierre Birkui, and Jean Luc Chassé. Modeling of Cardiac Electrophysiological Mechanisms : from Action Potential Genesis to its Propagation in Myocardium. Critical Reviews in Biomedical Engineering, 24:141-221, 1996.

[2] Y. Bourgault, M. Ethier, and V.G. LeBlanc. Simlulation of electrophysiological waves with an unstructured finite element method. M2AN, 2003.

[3] N.F. Britton. Reaction-diffusion equations and their applications to biology. London etc.: Academic Press, 1986.

[4] Haïm Brézis. Analyse fonctionnelle. Théorie et applications. Masson, 1983.

[5] Colli-Franzone, L. P. Guerri, C. Viganotti, E. Macchi, S. Baruffi, S. Spaggiari, and B. Taccardi. Potential fields generated by oblique dipole layers modeling excitation wavefronts in the anisotropic myocardium. Circ. Res., 51:330-346, 1982. 
[6] Robert Eymard, Thierry Gallouët, and Raphaèle Herbin. Finite volume methods. Ciarlet, P. G. (ed.) et al., Handbook of numerical analysis. Vol. 7, 2000.

[7] R. Fitzhugh. Impulses and physiological states in theoretical models of nerve membrane. Biophys. J., 1:445-465, 1961.

[8] Piero Colli Franzone and Giuseppe Savaré. Degenerate evolution systems modeling the cardiac electric field at micro- and macroscopic level. In Evolution equations, semigroups and functional analysis. Lorenzi, Alfredo (ed.) et al., In memory of Brunello Terreni, 2002.

[9] C.S. Henriquez. Simulating the electrical behaviour of cardiac tissue using the bidomain model. Crit. Rev. Biomed. Engr., 21:1-77, 1993.

[10] Dan Henry. Geometric theory of semilinear parabolic equations. SpringerVerlag, LNM 840, 1981.

[11] A.L. Hodgkin and A.F. Huxley. A quantitative description of membrane current and its application to conduction and excitation in nerve. J. Physiol., 117:500-544, 1952.

[12] J.P. Keener and J. Sneyd. Mathematical Physiology. Springer-Verlag, 1998.

[13] G.T. Lines, P. Grottum, A.J. Pullan, J. Sundes, and A. Tveito. Mathematical models and numerical methods for the forward problem in cardiac electrophysiology. Comput. Visual. Sci, 5:215-239, 2003.

[14] G.T. Lines, P. Grottum, and A. Tveito. Modeling the electrical activity of the heart: a bidomain model of the ventricles embedded in a torso. Comput. Viual. Sci., 5:195-213, 2003.

[15] J.L. Lions. Quelques méthodes de résolution des problèmes aux limites non linéaires. Dunod, 1969.

[16] J.S. Nagumo, S. Arimoto, and S. Yoshizawa. An active pulse transmission line simulating nerve axon. Proc. IRE, 50:2061-2071, 1962.

[17] M.P. Nash and Panfilov A.V. Electromechanical model of excitable tissue to study reentrant cardiac arrythmias. Progress in Biophysics \& Molecular Biology, 85:501-522, 2004.

[18] Alexander V. Panfilov and Rubin R Aliev. A simple two-variable model of cardiac excitation. Chaos Solitons and Fractals, 7(3):293-301, 1996.

[19] R.C. Penland, D.M. Harrild, and C.S. Henriquez. Modeling impulse propagation and extracellular potential distributions in anisotropic cardiac tissue using a finite volume element discretization. Computing and Visualization in Sciences, 4:215-226, 2004.

[20] S. Sanfelici. Convergence of the galerkin approximation of a degenerate evolution problem in electrocardiology. Numer. Methods for Partial Differential Equations, 18:218-240, 2002.

[21] M. Sermesant, Y. Coudière, H. Delingette, and N. Ayache. Progress towards an electromechanical model of the heart for cardiac image analysis. In IEEE International Symposium on Biomedical Imaging (ISBI'02), 2002.

[22] M. Sermesant, O. Faris, F. Evans, E. McVeigh, Y. Coudière, H. Delingette, and N. Ayache. Preliminary validation using in vivo measures of a macroscopic electrical model of the heart. In International Symposium on Surgery Simulation and Soft Tissue Modeling (IS4TM’03), 2003. 
[23] A.A. Shcherbakov. On the estimate of the attractor's dimension for multidimensional FitzHugh-Nagumo equations. Russ. J. Math. Phys., 1(4):533-538, 1993.

[24] Joel Smoller. Shock waves and reaction-diffusion equations. Springer- Verlag., 1983.

[25] Roger Temam. Infinite-dimensional dynamical systems in mechanics and physics. 2nd ed. Applied Mathematical Sciences. 68. New York, NY: Springer., 1997.

[26] A.D. Waller. A demonstration on man of electromotive changes accompanying the heart's beat. J. Physiol., 8:229-234, 1887. 